\title{
Accompagner de futurs traducteurs dans l'exploration des langues de spécialité, par temps de Covid-19
}

Helping translator trainees explore specialised languages during the Covid-19 crisis

Geneviève Bordet

\section{CpenEdition}

\section{Journals}

Édition électronique

URL : http://journals.openedition.org/asp/6528

DOI : 10.4000/asp.6528

ISSN : 2108-6354

\section{Éditeur}

Groupe d'étude et de recherche en anglais de spécialité

\section{Édition imprimée}

Date de publication : 1 novembre 2020

Pagination : 19-29

ISSN : 1246-8185

\section{Référence électronique}

Geneviève Bordet, «Accompagner de futurs traducteurs dans l'exploration des lanques de spécialité, par temps de Covid-19», ASp [En ligne], 78 | 2020, mis en ligne le 01 novembre 2020, consulté le 06 mars 2021. URL : http://journals.openedition.org/asp/6528 ; DOI : https://doi.org/10.4000/asp.6528

Ce document a été généré automatiquement le 6 mars 2021.

Tous droits réservés 


\title{
Accompagner de futurs traducteurs dans l'exploration des langues de spécialité, par temps de Covid-19
}

Helping translator trainees explore specialised languages during the Covid-19 crisis

\author{
Geneviève Bordet
}

\section{Avant-propos}

1 Ce compte rendu tire parti de l'actualité pour adapter une étape essentielle du métier de traducteur, la recherche terminologique, et ce dans une optique de formation de traducteurs mais qui pourrait être élargie à toute étude sur les discours spécialisés. L'auteur met en place un dispositif pédagogique qui doit tenir compte de contraintes extérieures inédites: la crise sanitaire que nous traversons impose le passage à un enseignement à distance.

Geneviève Bordet décrit le module intitulé «Atelier mémoire : recherche documentaire » qu'elle a déployé pour des étudiants de master 1 ILTS (Industrie des langues et traduction spécialisée) à l'Université de Paris et qui vise à les aider à l'élaboration de leur projet de mémoire de master 2. Cette démarche s'inscrit dans l'évolution du marché de la traduction: on recherche désormais moins de simples traducteurs que des langage analysts, présentant des compétences de localisation et de post-édition (Robert 2010). On peut rapprocher cette idée de la notion de "consultant en ingénierie linguistique» (Perrigaud 2014) doté de soft skills, ou compétences interpersonnelles, toujours plus nombreuses (relation client, savoir-être professionnel, etc.).

L'auteure propose une approche dynamique des langues de spécialité qui couple diverses méthodes (recherche documentaire, terminologie et analyse de corpus) et techniques mêlant notamment la technique dite du "QQQOCP » (5 Ws and H), la grille dite PPS (Position, Problem, Solution), le diagramme de Venn et la comparaison terminologique entre Google et Google Scholar. Les étudiants appliquent ces grilles d'analyse pour affiner leur problématique et en rendent compte en classe virtuelle sur la plateforme Renater. 
L'auteure tire un bilan positif de ce module qui a clairement renforcé la motivation des étudiants en cette période anxiogène, mais la lourdeur d'un système tout en distanciel l'amène à privilégier à l'avenir un dispositif hybride, couplant réalisation en salle de cours et système de remédiation individuelle en ligne. (Franck Barbin, Université Rennes 2)

\section{Introduction}

5 Le dispositif pédagogique dans lequel s'inscrit le module présenté ici peut difficilement être qualifié d'innovation puisque, créé il y a trente ans, il constituait déjà un élément structurant du master Industrie des langues et traduction spécialisée mis en place à l'UFR Études interculturelles et langues appliquées (EILA) de l'Université Paris Diderot (dorénavant Université de Paris) à l'initiative de Claudie Juilliard. Pourtant, l'originalité de ce dispositif, ses évolutions constantes, en font une réelle pratique innovante à plusieurs titres: mise en œuvre de l'interdisciplinarité dans l'approche de la langue de spécialité, réflexion sur la complémentarité avec la formation professionnelle dans ce master en alternance, puis intégration dans le dispositif d'experts spécialisés et de traducteurs et terminologues professionnels.

6 Le module que je présente dans ce compte rendu est par ailleurs nouveau à double titre : si le projet répond à la réforme récente qui vise à renforcer la continuité entre les deux années de master ${ }^{1}$, il a dû s'adapter à des circonstances sanitaires inédites résultant dans la mise en place en quelques jours d'un dispositif d'enseignement à distance.

7 Après avoir exposé le contexte et les objectifs du dispositif général, j'analyse les apports de ce module à la compréhension, par de futurs professionnels de la communication, de ce qui fonde l'identité des langues de spécialité à travers leur diversité.

\section{Contexte pédagogique}

Le module intitulé « Atelier mémoire : recherche documentaire » concerne trente-deux étudiants de première année de master inscrits en master Industrie des langues et traduction spécialisée (ILTS) à l'Université de Paris. Les étudiants sont répartis en deux groupes qui bénéficient chacun de deux heures de Travaux Dirigés (TD) toutes les deux semaines, durant le second semestre, soit douze heures au total pour chaque groupe. Le module fait partie de l'Unité d'enseignement (UE) «Connaissances appliquées à la traduction "; il est complété par un module "Atelier mémoire : terminologie " organisé en parallèle.

9 Les groupes ont été constitués en fonction de la seconde langue étrangère des étudiants, qui doivent faire preuve d'une excellente maîtrise du français et de l'anglais. Cette langue étrangère peut être l'espagnol ou l'allemand. Cependant, nombre d'étudiants viennent de l'étranger, notamment par l'intermédiaire de Campus France, et disposent également de leur langue maternelle : pour cette année 2019-2020, le chinois, l'italien, l'arabe et le russe. Ces étudiants peuvent choisir d'inclure leur langue maternelle comme langue cible pour le mémoire.

10 Le module devait se dérouler en présentiel, dans une salle de cours équipée d'ordinateurs au Centre de ressources en langues. Nous avons basculé vers une 
formation à distance à partir de la mi-mars jusqu'à la fin du semestre, examens compris.

\section{Des objectifs d'apprentissage et de production}

11 L'objectif de ce module est d'accompagner les étudiants de M1 dans le choix et l'élaboration de leur projet de mémoire, projet qui sera réalisé durant la seconde année. Si les étudiants ont passé avec succès divers tests de langues, certains ont une formation commerciale, juridique, scientifique ou technique. Il s'agit pour ces derniers d'une première confrontation avec les langues comme terrain de réflexion.

Tous devront maîtriser, au début de la seconde année, les connaissances de base indispensables pour la réalisation d'un projet de mémoire ambitieux, notamment en matière de recherche documentaire, terminologie et analyse de corpus. Il s'agit en effet de réaliser un travail de recherche et d'application centré sur la découverte de l'univers discursif d'un domaine de spécialité à travers ses caractéristiques terminologiques (Bordet 2013) et phraséologiques (Pecman \& Gledhill 2018). L'exploration du domaine s'appuie sur la maîtrise des outils d'information et de l'analyse de corpus (Kübler \& Volanschi 2012). Elle est appliquée à la traduction d'un texte du domaine (Froeliger 2013).

Les productions attendues des étudiants sont au nombre de trois :

1. Un commentaire écrit présente le domaine, décrit la démarche de recherche et le corpus, analyse les caractéristiques terminologiques spécifiques de ce domaine, puis argumente les choix de traduction.

2. Les résultats de recherches terminologiques, validés par un ou des experts du domaine, sont intégrés dans une base de données multidisciplinaire accessible en ligne, ARTES (Kübler \& Pecman 2012) et présentés sous la forme d'une arborescence sémantique (Bordet 2014).

3. Enfin, le mémoire comprend la traduction alignée d'un texte spécialisé d'un minimum de 20 000 signes. Ce texte doit n'avoir jamais été traduit et l'étudiant doit obtenir l'autorisation de son ou de ses auteurs ${ }^{2}$. Le mémoire dans son ensemble représente un tiers des ECTS attendus, les deux autres tiers étant attribués aux travaux liés aux cours et à la note attribuée par le tuteur en entreprise responsable de l'alternance. Ces trois notes ne sont pas compensables, ce qui donne au mémoire une importance décisive.

14 Jusqu'en 2018, ce travail était accompli exclusivement durant l'année de M2, les candidats étant admis essentiellement au niveau M2. En raison de la réforme des masters intervenue en 2016, le recrutement se fait maintenant très majoritairement à l'entrée en première année de master, même si quelques places ont été réservées pour permettre à d'éventuels candidats à la reconversion d'entrer directement en seconde année. De ces changements a résulté une nouvelle maquette de formation dont l'objectif central est d'assurer cohérence et continuité entre les deux années du diplôme. C'est la raison pour laquelle les deux " ateliers mémoire » (recherche documentaire et terminologie) ont été créés. Par ailleurs, ces deux ateliers étaient l'occasion d'alléger l'année de master 2, souvent considérée comme trop lourde, et d'améliorer le suivi individuel dans le choix du sujet de mémoire. 


\section{Une démarche interdisciplinaire}

15 La démarche adoptée pour l'ensemble du mémoire repose sur une complémentarité des connaissances théoriques requises pour les métiers liés au traitement de l'information multilingue car on trouve de plus en plus souvent des propositions de postes non pour des traducteurs, mais pour des « language analysts» ce qui englobe diverses fonctions nouvelles telles que « localisateur » ou " post-éditeur » (Robert 2010) ou encore ceux qu'au Québec on désigne comme « langagiers » (Sénécal 2010). Ces évolutions rendent encore plus nécessaire l'interdisciplinarité de la formation, qui allie linguistique de corpus, terminologie, traductologie, ainsi que recherche et structuration de l'information.

16 Le module "Atelier mémoire ", dans sa double déclinaison en terminologie et recherche et structuration de l'information, a été introduit au second semestre de la première année pour permettre aux étudiants de mieux percevoir la complémentarité de ces approches du discours spécialisé. Il s'agit de faire appréhender un domaine non comme une liste de termes et de concepts à maîtriser, mais comme une "langueculture " (Petit 2002), reflet des préoccupations d'une communauté de spécialistes réunis par une même problématique (Dressen-Hammouda 2003). En effet, la conception de la langue de spécialité qui sous-tend l'accompagnement de ces mémoires est celle d'une communauté, non pas réduite aux spécialistes d'un seul domaine de connaissances, mais composée d'acteurs réunis autour de questions communes tel que " comment lutter contre la propagation des virus » ou " quel est l'impact du changement climatique sur les conflits hommes - animaux » pour prendre des sujets proposés par les étudiants de M1 cette année.

17 Cette démarche très ouverte, qui permet une approche dynamique des langues de spécialité, peut paraître complexe et surtout insécurisante à des étudiants habitués à répondre à des consignes ou à traiter des sujets tels qu'ils ont été pensés par l'enseignant. Le fait de devoir élaborer soi-même la problématique que l'on va traiter, en s'appuyant sur un corpus que l'on constitue au fur et à mesure de cette élaboration, déroute souvent les étudiants. C'est la raison pour laquelle il nous a semblé nécessaire de renforcer l'accompagnement pour l'étape cruciale du choix du sujet et de sa transformation en une problématique.

18 Avant le confinement, j'avais largement insisté sur les interactions entre les étudiants et avec l'enseignante, avec notamment un travail en binôme où chacun se présentait à l'autre et exposait ses pistes de sujet. Puis un des membres du binôme exposait au groupe le résultat de cet entretien. Cette forme d'interaction a contribué à la mise en place de relations personnalisées, ce qui s'est avéré précieux lorsqu'il a fallu passer d'un jour à l'autre à l'enseignement à distance. Par la suite, l'envoi hebdomadaire de travaux individuels d'application au sujet choisi par chaque étudiant des outils vus en cours a encore renforcé la personnalisation du suivi.

19 Chaque séance de cours, d'abord en salle informatique, puis sur la plateforme Renater ${ }^{3}$, a permis d'introduire des outils méthodologiques et des ressources documentaires numériques nécessaires à l'appréhension d'un domaine de spécialité inconnu. Pour affiner la définition de leur sujet, les étudiants sont d'abord invités à le formuler sous forme de mots-clés et d'une question qui leur parait centrale. Ainsi, une étudiante, intéressée par les « boîtes noires » des avions, propose la question suivante : « Jusqu'à quel point peut-on croire à la fiabilité des boîtes noires? ». Après un cours qui porte sur 
les moteurs et les équations de recherche, les étudiants cherchent ensuite à élaborer des équations de recherche efficaces et comparent les résultats obtenus avec Google et Google Scholar, ce qui met en évidence la différence de discours entre langue générale et langue spécialisée (voir annexe 1). A cette occasion, une étudiante qui a choisi de travailler sur les « écomatériaux » note que si, avec Google, l'on trouve majoritairement ce terme dans des sites promouvant la construction durable, les documents scientifiques proposés par Google Scholar privilégient le terme " matériaux biosourcés ", moins évocateur, mais plus précis.

Dans une autre étape, sont abordées deux techniques pour aborder un sujet, notamment utilisées pour la formation des journalistes. La première est celle dite du " QQQOCP " ou en anglais " 5 Ws and $H$ » (Lasswell 1948). Cette technique consiste à questionner un sujet sous tous ses angles, comme dans cet exemple :

Qui est concerné par l'utilisation des boîtes noires, Quoi : de quoi est composé cet objet? Quand : depuis quand existent-elles ? Comment fonctionnent-elles? Pourquoi les utilise-t-on?

21 La deuxième technique, également anglo-saxonne, vise à élaborer une problématique dynamique. Utilisée, tout comme la précédente, pour la formation des journalistes, elle applique la grille dite PPS (Position, Problem, Solution) pour décrire une situation et en dégager une problématique, par exemple :

Position : les boîtes noires sont utilisées pour reconstituer les circonstances d'un accident aéronautique ; Problem : elles ne sont pas toujours fiables; Solution : analyse des causes et des solutions proposées pour améliorer leur fiabilité.

22 La problématique peut être plus complexe et plus difficile à dégager. Une étudiante, impressionnée par sa lecture récente du roman La panthère des neiges de Sylvain Tesson, souhaite travailler sur les conflits entre hommes et animaux dans un contexte de changement climatique. Ses premiers travaux de recherche lui permettent de proposer la formulation suivante :

Position: Hommes et animaux doivent coexister sur les mêmes territoires

Problem : Le changement climatique réduit la biodiversité animale, contribuant à

faire disparaître les proies traditionnelles des prédateurs, lesquels tendent à

s'attaquer aux troupeaux des hommes

Solution: Les scientifiques cherchent à élaborer des solutions pour renforcer la

biodiversité et lui permettre de s'adapter au changement climatique

Ces grilles d'analyse sont appliquées dans un travail individuel remis par courriel et sur Moodle. Elles sont reprises et discutées lors du cours à distance, la plateforme permettant de partager le document pour qu'il soit visible par tous. Les étudiants peuvent demander à intervenir pour des suggestions en utilisant l'icône « main » ou en écrivant à tous dans l'espace « chat ».

L'approche est ensuite élargie aux connaissances connexes liées à un domaine d'activité. Je propose l'utilisation d'un diagramme de Venn (voir annexes 2 et 3 ) pour représenter les divers domaines de connaissances et la manière dont ils se croisent dans une même problématique. C'est ainsi qu'une étudiante, qui s'intéresse à l'impact de la musique sur le cerveau, passe d'un premier schéma très simple qui croise musicologie, neurosciences et neurologie, à un schéma beaucoup plus élaboré de l'imbrication des disciplines et des connaissances nécessaires à l'étude de ce sujet.

Un autre étudiant propose de traiter des systèmes de datation en archéologie, ce qui l'amène à découvrir le domaine de l'archéométrie, qu'il définit ainsi : 
Les méthodes (de l'archéométrie) comprennent des études de sédimentologie, botanique, archéozoologie, anthropologie, analyses des matériaux dans un objectif d'interprétation et de datation des objets et sites archéologiques. suivant est essentiel pour le choix du sujet de mémoire : vérifier la possibilité d'appliquer à ce sujet une approche fondée sur l'observation de corpus comparables dans la langue source et dans la langue cible. C'est dans cette perspective que sont présentées les ressources numériques accessibles grâce à la plateforme de la bibliothèque universitaire, fermée depuis le début du confinement. Dans ce contexte, plusieurs grands éditeurs scientifiques ont libéré l'accès à un grand nombre de livres électroniques, ce qui, d'une part, fournit des ressources supplémentaires et, d'autre part, permet d'aborder de manière concrète les enjeux du libre accès à l'information scientifique et technique dans un contexte dématérialisé. Les étudiants doivent vérifier qu'il existe un nombre de publications récentes et accessibles suffisant pour la constitution d'un corpus représentatif du domaine abordé. L'objectif n'est pas simplement quantitatif. Il faut également veiller à ce que le corpus constitué reflète la diversité des genres textuels et documentaires qui caractérisent la production du domaine, qu'il soit technique ou scientifique.

\section{L'adaptation pédagogique suite au confinement}

Lobligation dans laquelle je me suis trouvée de passer d'une pédagogie classique à une pédagogie à distance ne paraît pas avoir nui à la réussite de la démarche, mais elle a certainement modifié ma relation aux étudiants. La fonctionnalité de partage d'écran, offerte par la plateforme, complétée par la possibilité d'intervention sur le clavardage (plus communément nommé " chat ») permet de faire des démonstrations efficaces. Elle permet aussi de donner à l'étudiant la possibilité d'exposer ses propres recherches. Pour renforcer le lien et l'interactivité, Je demandais aux étudiants qui prenaient la parole de mettre en route leur vidéo, parfois désactivée pour économiser la bande passante et éviter les coupures. De même, toutes les questions écrites sur le chat ont été lues à voix haute par l'enseignant qui indiquait le nom de leur auteur.

Cependant, les limites de temps ne permettant pas de donner la parole à tous, le rendu hebdomadaire de travaux pratiques commentés par écrit par l'enseignant s'est avéré indispensable pour repérer les étudiants en difficulté et leur proposer des pistes de travail.

$\mathrm{Au}$ fur et à mesure, se sont ajoutés, en fonction des besoins, des entretiens individuels proposés en fonction de la qualité des travaux pratiques rendus ou des échanges lors des visioconférences ${ }^{4}$. Il pouvait également s'agir de rares étudiants ne s'étant pas connectés aux séances. Je reviendrai sur ce point dans la section 6 . La mise en place d'une rubrique Questions $(\mathrm{FAQ})$ sur Moodle, élaborée à partir des échanges enregistrés sur le chat, n'a pas connu de réel succès, les étudiants préférant manifestement les échanges directs avec l'enseignante par courriel. 


\section{Critères et modalités d'évaluation des acquis d'apprentissage} aux futurs directeurs de mémoire. Il est donc défini comme un document de communication dont l'objectif est de convaincre le futur directeur ou la future directrice de l'intérêt du domaine choisi et de l'approche proposée. Constitué à partir des éléments d'information recueillis lors des travaux pratiques, il doit comprendre une présentation du domaine de spécialité proposé, du corpus bilingue comparable déjà constitué et une proposition de texte à traduire. Le dossier peut être rédigé en anglais ou en français, au choix de l'étudiant. Il est d'abord remis par courriel et sur Moodle, puis présenté lors d'un oral individuel pour lequel on a utilisé l'outil de visioconférence Skype, plateforme qui ne nécessite pas de réservation préalable et qui permet également le partage d'écran. Nous envisageons pour l'an prochain la mise en place d'une séance collective où chaque étudiant disposerait de dix minutes pour présenter son projet de mémoire dans un PowerPoint, ce qui permettrait à tout le groupe de bénéficier d'une vision d'ensemble des sujets traités, et faciliterait par la suite une atmosphère collaborative entre les étudiants.

Les critères d'évaluation mettent l'accent sur la clarté et la précision de la présentation de la problématique et du domaine choisi ainsi que la qualité de la rédaction. Pour l'oral, s'ajoute la capacité à prendre en compte les observations et à y répondre.

En fonction de l'épreuve orale, le dossier a parfois fait l'objet de modifications ou de compléments avant acceptation définitive. L'objectif est en effet de fournir au ou à la futur(e) directeur(trice) de mémoire un argumentaire recevable.

\section{Bilan de l'action de formation}

Le bilan de l'action est très largement positif. Un seul étudiant a abandonné pour des raisons sociales et familiales. Tous les étudiants ont rendu leur dossier, dans les délais demandés ou avec un léger aménagement. La situation exceptionnelle a certainement joué un rôle dans la forte motivation et l'assiduité des étudiants, dont un quart arrivait de l'étranger. Les dossiers sont, dans l'ensemble, d'une grande qualité dans la mesure où chacun a été en mesure de proposer un domaine de spécialité et de déterminer les types de connaissances requis pour sa compréhension. Ils ont également su proposer une amorce de corpus comparable convaincante sur le plan quantitatif comme sur le plan qualitatif, en raison de la diversité des genres représentés. L'argumentaire joint aux propositions de textes à traduire dénote une bonne compréhension de ce que sont les caractéristiques textuelles caractéristiques d'un type de discours. Les cinq travaux pratiques rendus et leur évaluation, les nombreux entretiens individuels ont permis un véritable suivi, pourtant rendu difficile par la distance.

Cet aspect positif a aussi son revers pour l'enseignant. La gestion des courriels et du planning d'entretiens est lourde, les entretiens souvent très longs. Par ailleurs, les difficultés matérielles se sont fait fortement sentir. Il a souvent été nécessaire de couper caméra et microphone des auditeurs pour économiser la bande passante, ce qui ne favorise pas la communication. Les entretiens individuels notamment ont été l'occasion de mesurer l'importance de la fracture numérique tant du point de vue de 
l'habileté dans le maniement des outils que du point de vue de l'accès aux technologies. Nombre d'entretiens démarrés sur skype ont progressivement évolué vers des applications portables telles Whatsapp voire directement vers le téléphone. Ils ont aussi été l'occasion de constater de visu les difficultés sociales dans lesquelles se débattent certains étudiants, hébergés avec la famille dans une seule pièce, avec un accès limité à l'unique ordinateur. Dans un cas extrême, l'entretien a permis de se rendre compte qu'un étudiant s'était vu obligé d'utiliser une voiture pour pouvoir s'isoler, faute de domicile. Les solutions se sont avérées très limitées, en l'absence de bibliothèques ouvertes et de réponse des services sociaux de l'université. Cependant, la prise de conscience de ces difficultés a permis de mettre en place un réseau de solidarité.

\section{Conclusion} de l'étranger. Néanmoins, c'est un dispositif lourd et exigeant pour l'enseignant sous cette forme. La forme idéale semble être un dispositif hybride, composé de séances en salles de cours incluant pour partie la réalisation des travaux pratiques et recourant si nécessaire, comme système de remédiation, à des séances individuelles à distance.

\section{BIBLIOGRAPHIE}

BORDET, Geneviève. 2013. « Brouillage des frontières, rencontres des domaines : quelles conséquences pour l'enseignement de la terminologie et de la traduction spécialisée ». ASp 64, 95-115.

ASp, $78 \mid 2020$ 
DRESSEN-HAMMOUDA, Dacia. 2003. « Contributions of an integrated genre theory of text and context to teaching LSP ». ASp 39-40. DOI : 10.4000/asp.1306.

FROELIGER, Nicolas. 2013. Les noces de l'analogique et du numérique. Paris : Éditions des Belles Lettres. KÜBLER, Natalie \& Mojca PECMAN. 2012. “The ARTES bilingual LSP dictionary: from collocation to higher order phraseology”. In Electronic Lexicography, GRANGER, s. \& M. PAQUOT (Eds). Oxford: Oxford University Press, pp. 187-209

KÜBLER, Natalie \& Alexandra VOLANSCHI. 2012. "Semantic prosody and specialised translation, or how a lexico-grammatical theory of language can help with specialised translation". In BOULTON, A., S. CARTER-THOMAS \& E. ROWLEY-JOLIVET (Eds.), Corpus-Informed Research and Learning in ESP: Issues and Applications. Amsterdam \& Philadelphia : John Benjamins, 105-135.

LASSWELL, Harold D. 1948. "The structure and function of communication in society". The Communication of Ideas 37/1, 136-139.

PECMAN, Mojca \& Christopher GLEDHILL. 2018. "How trainee translators and their teachers deal with phraseological units in the ARTES database". Équivalences 45/1, 235-258.

PERRIGAUD, Anne-Charlotte. 2014. « Mémoire de traduction, TAO, post-édition et traitement automatisé de la langue : le traducteur en quête de sens... ». Tralogy II. Consulté le 15/ 9/2020 <http://lodel.irevues.inist.fr/tralogy/index.php?id=300>.

PETIT, Michel. 2002. «Éditorial », ASp 35-36, 1-2.

ROBERT, Anne-Marie. 2010. « La post-édition : l'avenir incontournable du traducteur? ». Traduire $222,137-144$.

SENÉCAL, André. 2010. «L'endogénisme linguistique au Québec ». L'Actualité Langagière 7/3, 29 $<$ https://www.btb.termiumplus.gc.ca/tpv2guides/guides/chroniq/index-eng.html? lang=eng\&lettr=indx_autr8cfx_HlRyOus\&page=9_aFmPCnr3UQ.html>.

\section{ANNEXES}

Annexe 1 : Premières équations de recherche et découverte de la diversité des ressources documentaires dans différentes cultures (Google Scholar)

\begin{tabular}{|l|l|l|l|}
\hline Langue & Formulation de recherche & Résultats & Année de publication \\
\hline Anglais & $\begin{array}{l}\text { Neural plasticity }+ \text { musical } \\
\text { training }\end{array}$ & 27600 & Des années 1950 à 2019 \\
\hline Français & Musique et plasticité cérébrale & 3510 & Des années 1950 à 2019 \\
\hline Chinois & 意乐+太璃可塑性 & 1620 & De 1997 à 2019 \\
\hline
\end{tabular}

Annexe 2 : Première version du diagramme de Venn 


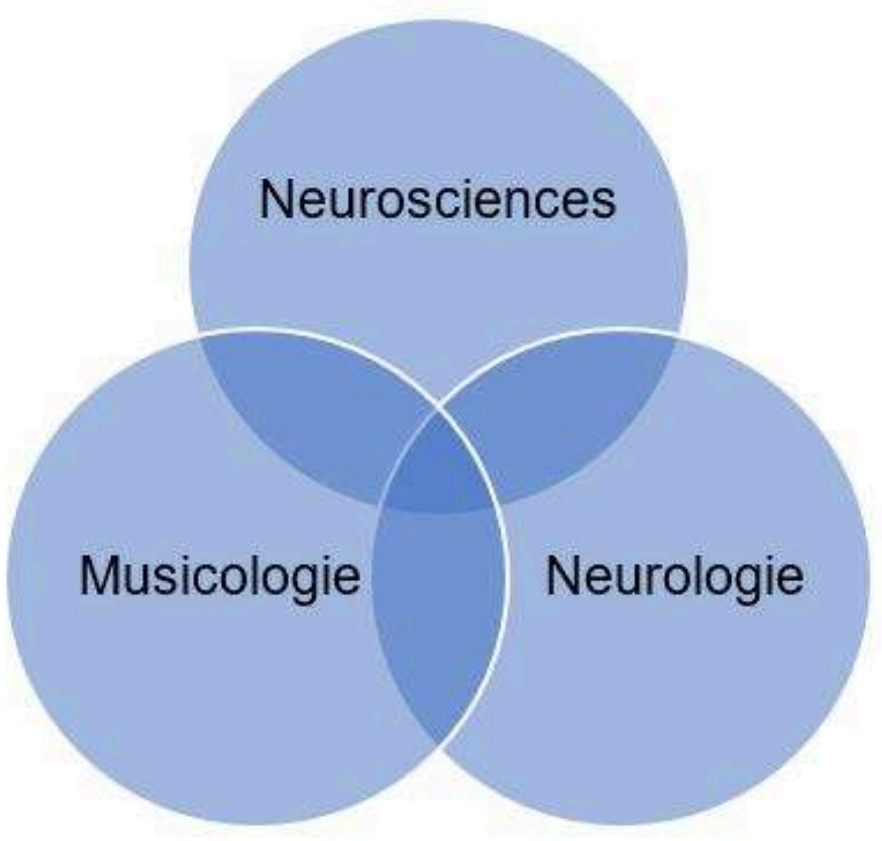

Annexe 3 : Version finale du diagramme de Venn

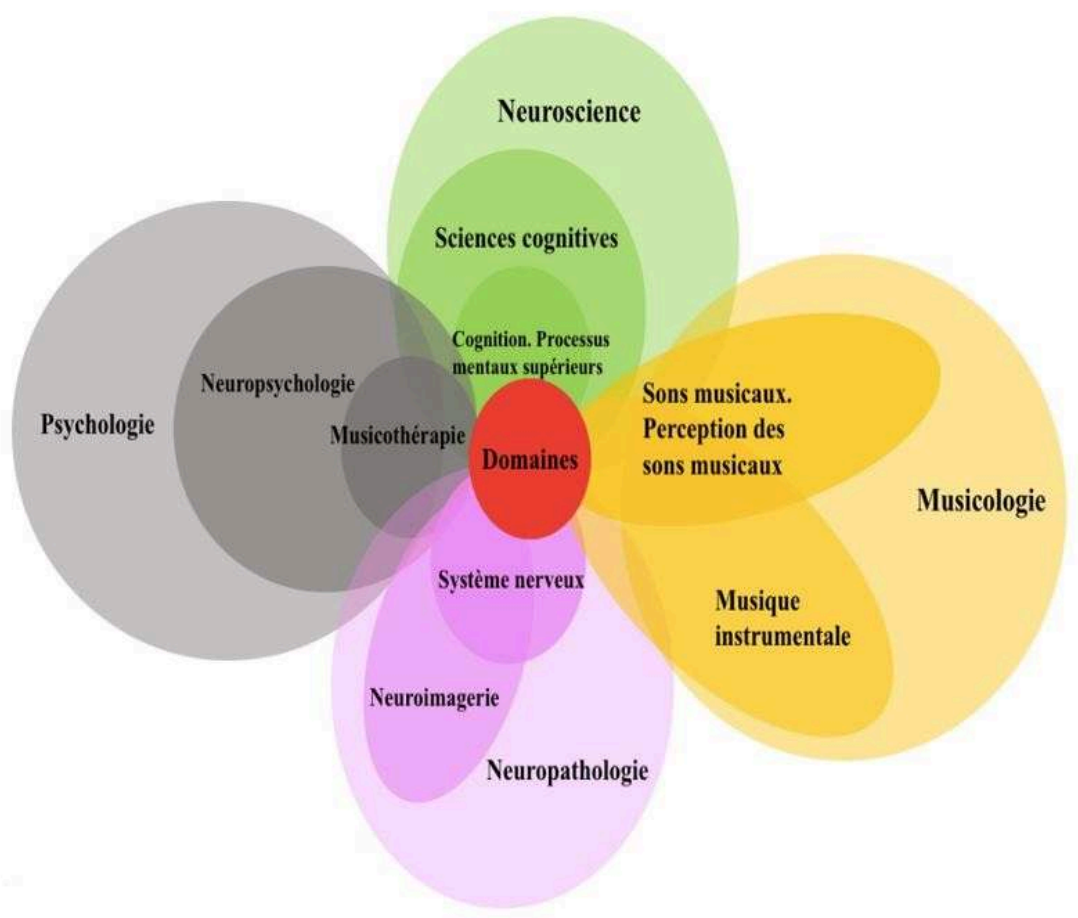

\section{NOTES}

1. Loi du 23 décembre 2016 portant adaptation du deuxième cycle de l'enseignement supérieur français au système Licence-Master-Doctorat. 
2. <https://www.eila.univ-paris-diderot.fr/formations-pro/masterpro/ilts/memoire>.

3. Réseau national de télécommunications pour la technologie, l'enseignement et la recherche $<$ https://rendez-vous.renater.fr/home/>.

4. L'outil utilisé pour ces entretiens était Skype.

\section{INDEX}

Mots-clés : enseignement à distance, langue de spécialité, mémoire de master, recherche documentaire, terminologie

Keywords : distance teaching, information retrieval, language for specific purposes, master's thesis, terminology

\section{AUTEUR}

\section{GENEVIÈVE BORDET}

Geneviève Bordet est maître de conférences en linguistique anglaise depuis huit ans à l'Université de Paris. Elle a codirigé le master de traduction spécialisée de 2012 à 2020. Un premier axe de ses recherches porte sur les mécanismes de construction d'une cohésion et d'une autorité scientifique dans les résumés de thèse, en comparant les stratégies en anglais des locuteurs natifs anglophones et francophones. Le deuxième axe s'appuie sur son expérience d'enseignante pour mettre en évidence les interactions entre recherche documentaire, terminologie et traduction dans la formation des traducteurs spécialisés. bordet@eila.univ-parisdiderot.fr 